\title{
1 Globale Erwärmung: Ist ein Kurswechsel möglich?
}

\author{
Volker Wittpahl
}

Im September 2015 haben die Vereinten Nationen die Ziele für eine nachhaltige Entwicklung verabschiedet, die bis zum Jahr 2030 erreicht werden sollen (United Nations 2015). Es wurden 17 Ziele der nachhaltigen Entwicklung, die "Strategic Development Goals (SDG)" , in einer Liste von 169 Zielen mit 232 einzelnen Indikatoren definiert, zu denen sich alle Länder der Welt verpflichtet haben.

Schaut man sich die Ziele im Detail an, so handelt es sich um eine Mischung aus gesellschaftlichen, Umwelt- und Klima-Zielen (Abb. 1.1). Selbst wenn alle Länder die Ziele engagiert angehen wollen, so haben sie mit einer großen Herausforderung bei ihrer Umsetzung zu kämpfen: Es gibt wechselwirkende Einflüsse, welche die Zielerreichung beeinflussen.

Um eine nachhaltige globale Entwicklung zu ermöglichen und Maßnahmen sowie ihre Wirkungen besser einordnen und abschätzen zu können, ist es deshalb notwendig, die Haupttreiber für negative Einflüsse und ihre Wechselwirkungen zu verstehen. Als ein solch starker Treiber lässt sich eindeutig die globale Erwärmung identifizieren.
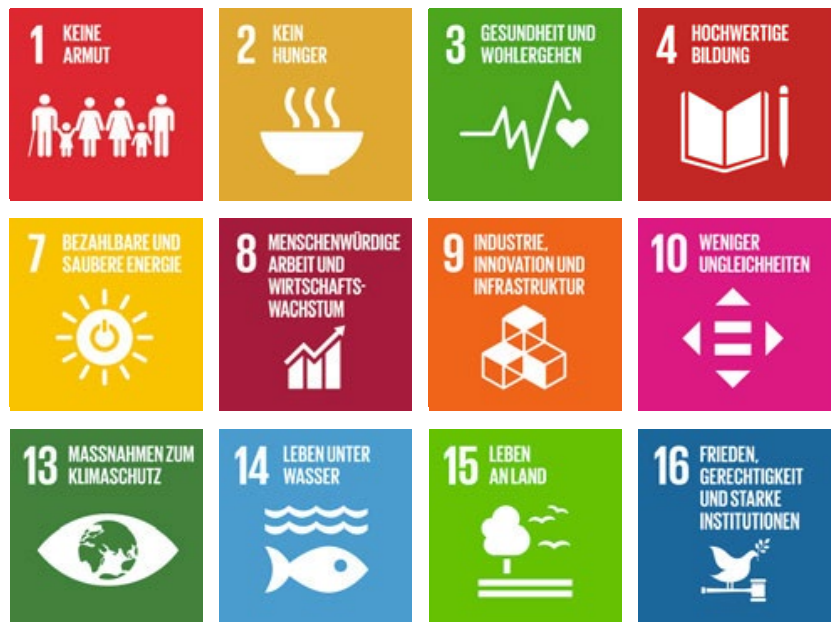
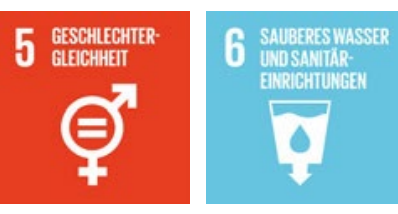
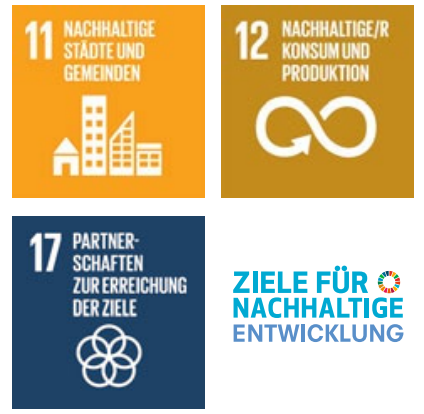

ZIELE FŮR NACHHALTIGE ENTWICKLUNG

Abb. 1.1 Nachhaltigkeitsziele der Vereinten Nationen. (Quelle: Bundesregierung 2020) 


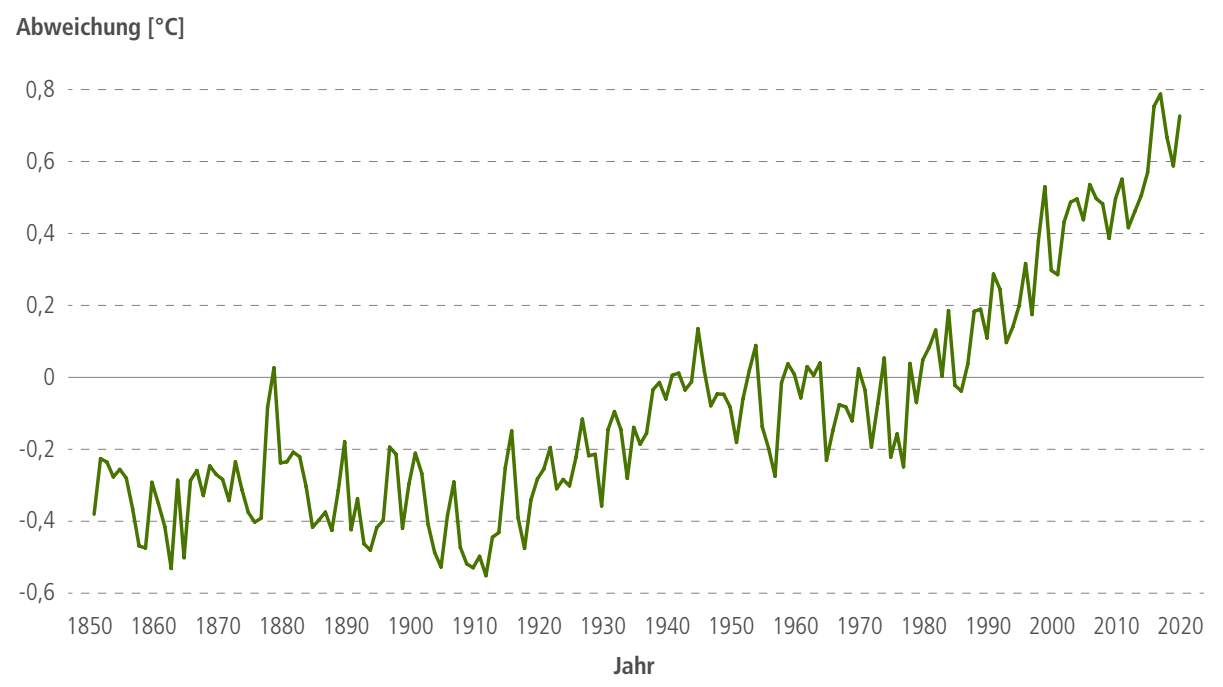

Abb. 1.2 Abweichung der globalen Mitteltemperatur: Alle 19 Jahre seit 2001 gehören zu den 20 wärmsten seit Beginn der Aufzeichnungen. (Eigene Darstellung nach Umweltbundesamt 2020a)

Das diffuse Gefühl, dass etwas im größeren Maßstab nicht mehr stimmt, hat sich in der Jugendbewegung der Fridays for Future Bahn gebrochen. Schüler:innen und Studierende fordern Entscheidungsträger:innen aus Politik und Wirtschaft zum Handeln auf, dessen Grundlage auf Ergebnissen der Wissenschaft fußen möge. Tatsächlich bedarf es eines besseren Grundverständnisses für die wesentlichen Zusammenhänge und unserer Handlungsoptionen, um Sachdiskussionen zu dem Themenkomplex Klima, Erderwärmung und ihren Folgen führen zu können, statt von Halbwissen geprägte emotionale Debatten.

\section{Die beschleunigte globale Erwärmung ist das aktuelle Haupt-Risiko}

Folgt man der Wissenschaft, zeigt sich, dass die Zahlen und Prognosen zur Erderwärmung in immer kürzeren Abständen nach oben korrigiert werden müssen. So gehören alle 19 Jahre seit 2001 zu den 20 wärmsten Jahren seit Aufzeichnung der Wetterdaten (Abb. 1.2, Faust et al. 2020).

Schon 2018 hat sich der Internationale Klimarat IPC ${ }^{1}$ von dem Ziel verabschiedet die Erderwärmung bis zum Jahr 2100 nicht über 1,5 Grad Celsius ansteigen zu las-

1 Englisch: Intergovernmental Panel on Climate Change (IPCC). 
sen: "Die globale Erwärmung ${ }^{2}$ erreicht $1,5^{\circ} \mathrm{C}$ wahrscheinlich zwischen 2030 und 2052, wenn sie mit der aktuellen Geschwindigkeit weiter zunimmt." (IPCC 2018:8)

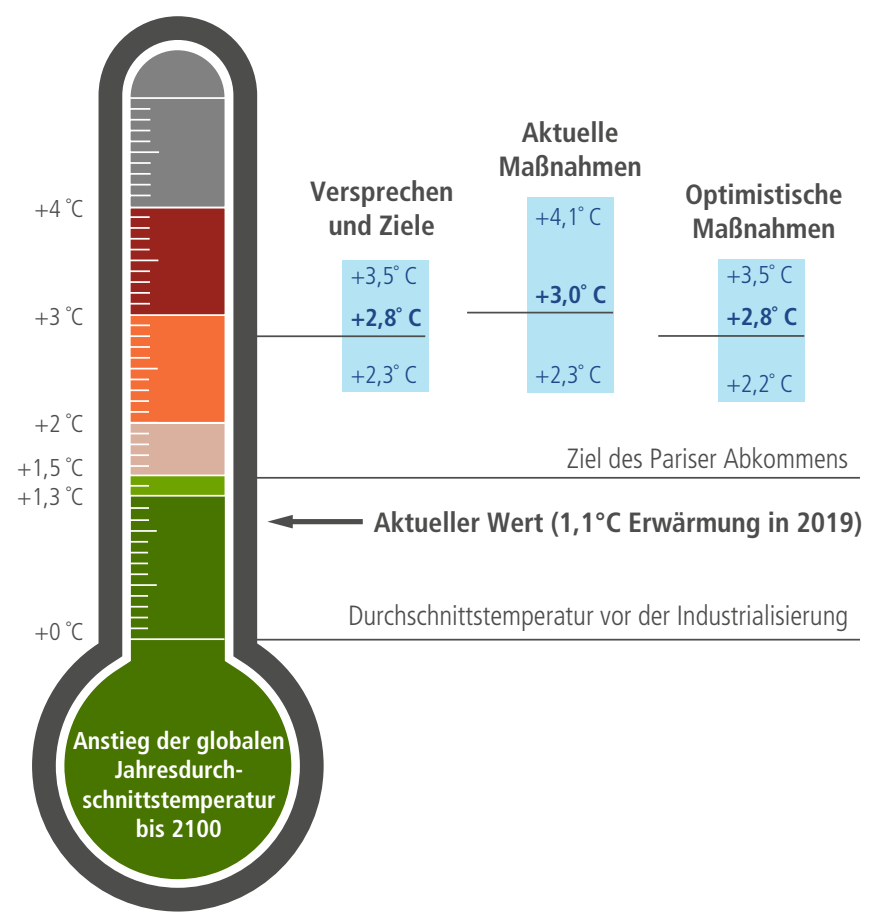

Abb. 1.3 Climate Action Tracker (CAT): Prognose des globalen Temperaturanstiegs bis 2100 basierend auf Zusicherungen und Zielen wie auch aktuellen Maßnahmen vom Dezember 2019. (Eigene Darstellung nach Climate Action Tracker 2019)

Laut der Weltorganisation für Meteorologie $(\mathrm{WMO})^{3}$ ist das globale Temperaturmittel gegenüber dem vorindustriellen Vergleichszeitraum zwischen 1850 und 1900 aktuell um 1,1 Grad Celsius gestiegen (WMO 2020a) und wird mit einer zwanzigprozentigen Wahrscheinlichkeit im Zeitraum bis 2024 auf 1,5 Grad Celsius ansteigen (WMO 2020b). In Deutschland wird laut Deutschem Wetterdienst (DWD) schon 2030 eine

2 Bezogen auf das Temperaturniveau von 1850 bis 1900.

3 Englisch: World Meteorological Organization (WMO). 
Erwärmung um zwei Grad Celsius gegenüber dem Bezugszeitraum von 1981 bis 2010 erreicht werden (DWD 2020).

Im Vergleich zu den Messungen der Meteorolog:innen sind in Abb. 1.3 die Prognosen des Climate Action Tracker (CAT) für die Durchschnittstemperaturen im Jahr 2100 angegeben, die sich aufgrund von internationalen Zielen und Versprechen sowie deren zugrundeliegenden Maßnahmen ergeben würden (Climate Action Tracker 2019).

Um eine Vorstellung zu entwickeln, was sich hinter der beschleunigten Erderwärmung verbirgt, ist es hilfreich, sich die Effekte vor Augen zu führen, die in Kaskaden mit dem Temperaturanstieg einhergehen. Da sind zunächst die globalen Kipp-Punkte, im Englischen Tipping Points. Dies sind Ereignisse, die ab einer bestimmten mittleren Erderwärmung einsetzen und sich dann nicht mehr stoppen oder umkehren lassen. Im Kap. 3 zu potenziellen Klimakonsequenzen sind globale Kipp-Punkte zusammengestellt. Neben diesen Kipp-Punkten lassen sich weitere Effekte als Kaskaden zur Temperaturerhöhung durchdeklinieren, die vor Augen führen, wie die Erderwärmung die verschiedenen Nachhaltigkeitsziele der Vereinten Nationen beeinflusst. Schlaglichtartig hier die exemplarische Darstellung einer Auswirkungskaskade:

Die globale Erwärmung führt zu ...

... Dürren, diese wiederum führen zu

- einem Rückgang von landwirtschaftlichen Nutzflächen. Zum Beispiel verdorren der Mittelmeerraum sowie weite Teile Indiens bei einer Erwärmung um 2 Grad Celsius (Wallace-Wells 2019:73),

- mit der Folge, dass weniger Anbauflächen mit weniger Erträgen zur Verfügung stehen, da sich mit jedem Grad Erwärmung die Ernteerträge um 10 Prozent mindern (Battisti et al. 2009), was wiederum zu einer

- Verknappung von Lebensmitteln führt.

- Flächenbränden und

- der Reduzierung von $\mathrm{CO}_{2}$-speichernden Pflanzen

- mit der Folge eines Rückkopplungseffekts durch weniger Kohlenstoffsenken, der den Temperaturanstieg beschleunigt.

- Verknappung von Süßwasser wodurch

- die Klima-Migration zunimmt. 
... Regionen, in denen es lebensgefährlich wird, sich im Sommer draußen aufzuhalten ${ }^{4}$, was zum Beispiel für Regionen im Nahen Osten und in Indien bei einer Erwärmung um 2 Grad Celsius vorhergesagt wird (Wallace-Wells 2019:56),

- mit Folge einer globalen Reduktion von urban nutzbaren Flächen, wodurch wiederum

- die Klima-Migration zunimmt.

- erhöhten Gesundheitsrisiken in Europa wie

- der Risikozunahme von hitzebedingten Erkrankungen und Todesfällen (World Health Organization 2017:7),

- dem Aufkommen von Tropenkrankheiten in Mitteleuropa (World Health Organization 2017:40 f.) und

- den potenziellen Risiken bei der Ernährung und der Ernährungssicherheit (World Health Organization 2017:46 f.), zu denen u. a.

- eine $\mathrm{CO}_{2}$-bedingte Abnahme von Nährstoffen wie Mineralien und Proteinen in den geernteten Lebensmitteln gehört ${ }^{5}$.

\section{Zwischenfazit}

Betrachtet man die bis hier umrissene Faktenlage zur Entwicklung der globalen Erwärmung, so drängen sich drei Schlussfolgerungen auf:

1. Die Erderwärmung nimmt trotz der Maßnahmen zu, welche die Staaten schon auf den Weg gebracht haben, und sie beschleunigt sich stärker als man in den Prognosen der vergangenen Jahre angenommen hat.

2. Die Effekte der Erderwärmung und ihrer Folgen werden inzwischen an vielen Stellen im Alltag bemerkbar, wobei die kausalen Zusammenhänge zwischen der Erderwärmung und dem Auftauchen von Effekten entlang der komplexen Auswrikungskaskaden sich nicht leicht erkennen lassen.

3. Die Effekte der beschleunigten Erderwärmung beeinflussen in einem nicht unerheblichen Umfang die Zielerreichung der SDG, wie man an der exemplarischen Auswirkungskaskade für die Ziele Kein Hunger (2), Gesundheit und Wohlerge-

4 Ein Drittel der heutigen Weltbevölkerung ist 20 Tage im Jahr einer tödlichen Hitzewelle ausgesetzt. Bei einer Erwärmung um $2{ }^{\circ} \mathrm{C}$ bis zum Jahr 2100 wird diese Zahl auf die Hälfte der Weltbevölkerung ansteigen (Wallace-Wells 2019:65).

5 Siehe auch Wallace-Wells 2019:75 $f$. 
hen (3) und Sauberes Wasser (6) leicht nachvollziehen kann. Dies bedeutet nicht, dass andere Effekte nicht auch bedeutenden Einfluss auf die Zielerreichung der SDG haben. Sie sind jedoch weniger komplex und einfacher zu verstehen als die Erderwärmung, welche das Ergebnis von unterschiedlichen, global miteinander wechselwirkenden Veränderungen des Klimas ist.

\section{Lässt sich die globale Erwärmung reduzieren oder umkehren?}

Das Klima ist ein komplexes System, das man sich als einen sehr großen Regelkreis vorstellen kann, in dem viele lokale und globale Einflussfaktoren miteinander wechselwirken. Die Erderwärmung ist in diesem System eine globale Größe, auf die eine Vielzahl von Einflussfaktoren einwirken, die über die ganze Erde verteilt sind und über eine Zeitspanne von einem Jahr gemessen werden müssen, um den Wert der globalen Erwärmung zu bestimmen und Änderungen zu vorhergehenden Jahren zu erfassen. Das unmittelbar zu verstehen, fällt uns Menschen schwer, denn wir nehmen Temperatur mit unseren Sinnen nur lokal und jeweils als absolute Größe wahr. Wir haben kein Temperaturgedächtnis. Es ist warm oder es ist kalt. Ob es gestern gegen 13 Uhr genauso warm war wie vor einer Woche um 13 Uhr, können wir ohne technische Hilfsmittel nicht sagen.

\section{Willkommen auf der MS Klima}

Die menschliche Wahrnehmung der Erderwärmung ähnelt dem Phänomen bei der Kursänderung eines Kreuzfahrtschiffes. Wenn der Kapitän auf der Brücke eine Kursänderung durchführt, so dauert es eine Weile und mehrere hundert Meter, bis ein Beobachter an der Reling diese Kursänderung wahrnimmt, da Referenzpunkte am Horizont oder in Schiffsnähe nur langsam ihre Position zueinander ändern. Die Erderwärmung bzw. die thermische Energiebilanz in der Erdatmosphäre repräsentiert in diesem Bild den Kurs, während das Schiff, die "MS Klima”, das komplexe KlimaSystem darstellen soll. Haupteinflussfaktoren und Auslöser für Kursänderungen, also Bewegungen am Steuerrad, sind trotz ihrer geringen Konzentration die Spurengase in der Atmosphäre wie Kohlendioxid $\left(\mathrm{CO}_{2}\right)$, Wasserdampf $\left(\mathrm{H}_{2} \mathrm{O}\right)$ oder Methan $\left(\mathrm{CH}_{4}\right)$. Gegenüber dem Stand von 1990 leisteten die langlebigen Treibhausgase im Jahr 2019 einen um 45 Prozent höheren Beitrag zur globalen Erwärmung (Umweltbundesamt 2020b), was im Bild der MS Klima einer massiven Kursänderung über einem längeren Zeitraum entspricht.

Die Ursachen und Wechselwirkungen für die globale Erwärmung verbergen sich hinter dem vereinfachenden Begriff des Treibhauseffekts. Wie Klima und Erderwärmung physikalisch funktionieren, wird ausführlicher im folgenden Kapitel dieses Buches beschrieben. Die physikalische Erklärung des Treibhauseffekts basiert auf der Energiebilanz der Atmosphäre. Das Einbringen von zusätzlichem $\mathrm{CO}_{2}$ in die Atmosphäre 
durch die Verbrennung fossiler Energieträger verändert die Lage des Energiegleichgewichts (Strahlungsbilanz), was zu einer Erhöhung der Lufttemperatur in Erdbodennähe führt. Die Konzentration des $\mathrm{CO}_{2}$ in der Luft wird als Teile pro Million Teilchen (Englisch: parts per million; ppm) angegeben und lag seit über einer halben Million Jahren nie über dem Wert von 300 ppm $^{6}$. Dies konnte man anhand von Messungen an Eiskernbohrungen aus den Polarregionen nachweisen. Mithilfe der Konzentrationsschwankungen kann man auch anhand von Gesteinsuntersuchungen erklären, wie sich das globale und lokale Klima über sehr lange Zeiträume verändert hat. Somit können Wissenschaftler:innen prinzipiell aus den Daten der Vergangenheit für $\mathrm{CO}_{2}-$ Konzentrationen bis 300 ppm validierbare Wechselwirkungsmodelle für das Klima entwickeln.

Während die $\mathrm{CO}_{2}$-Konzentration zur vorindustriellen Zeit um das Jahr 1800 einen Wert von 280 ppm aufwies, so hat sie im Jahr 2019 den Wert von knapp 410 ppm erreicht (Umweltbundesamt 2020b). Damit ist heute eine $\mathrm{CO}_{2}$-Konzentration weit jenseits des Bereichs gegeben, in dem sich das globale Klima über Jahrhunderttausende zwischen Warmzeiten und Eiszeiten bewegt hat.

Zu der aktuellen $\mathrm{CO}_{2}$-Konzentration gibt es keine Erfahrungswerte für eine Modellbildung. Sicher ist, dass es Wechselwirkungen gibt, die aufeinander rückkoppeln und ihren Einfluss gegenseitig verstärken werden. Unbekannt ist aber, wie sie sich wechselseitig beeinflussen. Laut dem internationalen Klimarat IPCC ist für eine $\mathrm{CO}_{2}-$ Konzentration um 450 ppm, das heißt von 430 bis 480 ppm, ein Temperaturanstieg von 2 Grad Celsius bis 4 Grad Celsius ${ }^{7}$ wahrscheinlich (IPCC 2014:88), verbunden mit dem Umstand, dass schon globale Kipp-Punkte ausgelöst werden. Mit einer $\mathrm{CO}_{2}-$ Konzentration von 500 ppm in der Atmosphäre geht eine Temperaturerhöhung von 3 bis 4 Grad Celsius ${ }^{8}$ einher (IPCC 2014:88), die mit der Passage weiterer globaler Kipp-Punkte verbunden ist.

Kursänderungen der MS Klima: $\mathrm{CO}_{2}$-Emissionen, $\mathrm{CO}_{2}$-Abscheidungen und Rückkopplungseffekte

Um die Energiebilanz der Atmosphäre wieder in einen Bereich zu bringen, für den ein Regelverhalten des Klimas bekannt ist, wird es nicht ausreichen, nur die $\mathrm{CO}_{2}$-Emissionen zu vermindern. Dies bestätigen auch Oliver Geden, Leitautor für den sechsten Sachstandsbericht des IPCC, und Felix Schenuit in ihrer im Mai 2020 erschienenen Studie "Unkonventioneller Klimaschutz", die einleitend beginnt: "Wenn die EU bis

6 Die Konzentration hat in den letzten 650.000 Jahren sich kaum geändert, sondern lediglich im Bereich von 180 bis 300 ppm variiert (Jalili et al. 2011:355).

7 Bezogen auf das Temperaturniveau von 1850 bis 1900.

8 Bezogen auf das Temperaturniveau von 1850 bis 1900. 
2050 Netto-Null-Emissionen erreichen will, wird es nicht genügen, konventionelle Klimaschutzmaßnahmen zur Emissionsvermeidung zu ergreifen. Um unvermeidbare Restemissionen auszugleichen, werden zusätzlich auch unkonventionelle Maßnahmen zur Entnahme von $\mathrm{CO}_{2}$ aus der Atmosphäre notwendig sein [...]." (Geden, Schenuit 2020:2)

Dass man neben der Reduktion von $\mathrm{CO}_{2}$-Emissionen auch der Atmosphäre $\mathrm{CO}_{2}$ entnehmen muss, sagt auch der Internationale Klimarat IPCC seit mehreren Jahren (IPCC 2014:66). Dies geht im Diskurs meist unter, da sich die Diskussionen in der Öffentlichkeit fast ausschließlich mit der Reduktion der $\mathrm{CO}_{2}$-Emissionen befassen.

Hinter dieser Aussage steht die Erkenntnis, dass selbst eine sofortige globale $\mathrm{CO}_{2}$ Null-Emission nicht ausreicht, um den Anstieg der globalen Erderwärmung aufzuhalten oder umzukehren. Die Erderwärmung wird abgeflacht fortschreiten, bis sich ein neues thermisches Gleichgewicht in der Atmosphäre einstellt, das mit der erhöhten $\mathrm{CO}_{2}$-Konzentration korreliert. In Analogie bedeutet dies, dass die MS Klima einen Punkt erreicht, an der sich das Schiff nicht mehr weiter vom ursprünglichen Kurs entfernt, aber inn auch nicht wieder einschlagen wird. Um auf eine $\mathrm{CO}_{2}$-Konzentration von 300 ppm oder sogar 280 ppm zurückzugelangen, muss neben der $\mathrm{CO}_{2}$-NullEmission in erheblichem Umfang das $\mathrm{CO}_{2}$ aus der Atmosphäre herausgewaschen werden. Zur $\mathrm{CO}_{2}$-Abscheidung und -Speicherung gibt es inzwischen unter dem Begriff "Carbon Capturing" Technologien, die mit ihrem Potenzial und aktuellen Entwicklungsstand ausführlicher im Kap. 6 "Vom Klimagas zum Wertstoff: $\mathrm{CO}_{2}$ " vorgestellt werden.

Es gibt immer wieder hoffnungsvolle Entwicklungen und Entdeckungen, wie zum Beispiel die "Beschleunigte Gesteinsverwitterung", bei der man Basaltstaub auf Äcker ausbringt. Damit lassen sich pro Jahr zwei Milliarden Tonnen $\mathrm{CO}_{2}$ aus der Atmosphäre entfernen, und dies wiederum entspricht einem Viertel der durch die Landwirtschaft erzeugten $\mathrm{CO}_{2}$-Emissionen, die sich so einsparen ließen (Beerling et al. 2020).

All diesen Technologien ist gemein, dass ihre Entwicklungen gerade erst am Anfang stehen und sie in den kommenden Jahren keinen kurzfristigen Beitrag zur Abschwächung oder Umkehr der Erderwärmung leisten werden.

Neben den $\mathrm{CO}_{2}$-Emissionen durch Verbrennung fossiler Energieträger kann die Erderwärmung zusätzlich durch Rückkopplungseffekte beschleunigt werden. So haben die Brände in Australien vom September 2019 bis zum Februar 2020 etwa 830 Millionen Tonnen zusätzliche $\mathrm{CO}_{2}$-Emission in die Atmosphäre eingebracht (Commonwealth of Australia 2020:9). Zum Vergleich: Der gesamte globale Flugverkehr im Jahr 2018 hat 918 Millionen Tonnen CO2- Emissionen verursacht (Graver, B et al. 2019). Damit wird deutlich, wie schnell sich die geplante Zielerreichung einer maximalen 
globalen $\mathrm{CO}_{2}$-Konzentration verschärfen kann; zur Kompensation der zusätzlichen $\mathrm{CO}_{2}$-Emissionen durch die australischen Flächenbrände müsste man zum Beispiel weltweit knapp ein Jahr auf das Fliegen verzichten. Dabei gab es neben den Flächenbränden in Australien zur gleichen Zeit auch noch Brände im Amazonasgebiet und in Russland, deren $\mathrm{CO}_{2}$-Eintrag damit noch nicht kompensiert wäre. Auch ist in dieser Kalkulation der Verlust an $\mathrm{CO}_{2}$-Senken in Form von Pflanzen und Bäumen noch nicht berücksichtigt. Ein weiterer Rückkopplungseffekt aus den Flächenbränden kann sein, dass das Eis an den Polkappen verrußt und somit weniger Sonnenlicht reflektiert wird: Die Albedo - also das Rückstrahlvermögen - reduziert sich und stattdessen wird das Sonnenlicht in Wärme umgewandelt, wodurch das polare Eis schneller schmilzt.

Neben dem Spurengas Kohlendioxid gibt es in der Atmosphäre weitere Spurengase, deren erhöhte Konzentrationen die Strahlungsbilanz ebenfalls zu Gleichgewichten bei höheren Bodentemperaturen treiben. So ist die Treibhauswirkung von $\mathrm{CH}_{4}$ gegenüber $\mathrm{CO}_{2}$ über einen Zeitraum von 100 Jahren um 28 Mal stärker und über 20 Jahre gerechnet sogar um 86 Mal stärker. US-Forscher haben in Studien im Jahr 2020 errechnet, dass der weltweite $\mathrm{CH}_{4}$-Ausstoß 2017 einen neuen Höchststand erreicht hat und mehr als die Hälfte davon durch Aktivitäten des Menschen verursacht war (Jackson et al. 2020 sowie Saunois et al. 2020). Der Ausstoß im Jahr 2017 hat gegenüber dem Durchschnitt der Jahre 2000 bis 2006 um neun Prozent zugenommen. Dazu sagt Pep Canadell vom "CSIRO Oceans and Atmosphere" im australischen Canberra: "Methan ist jetzt für 23 Prozent der globalen Erwärmung aufgrund von Treibhausgasen verantwortlich" (Tagesschau 2020).

Diese Werte gehen laut Forscher:innen einher mit Prognosen, die bis Ende des Jahrhunderts eine Temperaturerhöhung von drei bis vier Grad annehmen, und entsprechen damit der pessimistischsten Prognose des IPCC (Zeit 2020).

Eine Beschleunigung der Erderwärmung kann dazu führen, dass der Permafrost in Russland schneller auftaut als angenommen und zusätzliches Methan freigesetzt wird, das den Erwärmungsprozess durch Absorption in der Atmosphäre zusätzlich beschleunigt. Im Bild der MS Klima bedeutet dies, dass es neben dem großen Ruder $\mathrm{CO}_{2}$-Konzentration noch zusätzlich ein Bugstrahlruder $\mathrm{CH}_{4}$-Konzentration gibt, welches durch einen quer zum Schiff wirkenden Antrieb die Kursänderung zusätzlich verstärkt.

Die mittel- und langfristigen Rückkopplungseffekte durch die Ozeane, die Biosphäre und die Böden für sehr hohe $\mathrm{CO}_{2}$ - und $\mathrm{CH}_{4}$-Konzentrationen in der Atmosphäre sind bislang kaum bekannt. Gleiches gilt für den Einfluss von Wolken und Aerosolen auf das Klima. Auch ist offen, welche weiteren Rückkopplungen sich ergeben, wenn 
globale Kipp-Punkte passiert werden, die bei einer Erderwärmung von 2 Grad Celsius $^{9}$ erwartet werden.

Es lässt sich festhalten, dass es kein Modell für die nächsten Jahre gibt, das die zum Teil unbekannten Rückkopplungseffekte sicher einbeziehen kann.

Wohin geht die Reise der MS Klima?

Die Situation an Bord der MS Klima stellt sich derzeit wie folgt dar: Seit etwa 200 Jahren drehen unterschiedliche Akteure der Menschheit am Steuerrad für das Ruder $\mathrm{CO}_{2}$-Konzentration. Alle haben in dieser Zeit den Kurs in Richtung Erhöhung eingeschlagen, sodass man sich mit aktuell 410 ppm $\mathrm{CO}_{2}$ stark vom ursprünglichen Ausgangskurs 280 ppm $\mathrm{CO}_{2}$ entfernt hat. Inzwischen gibt es einige Eingriffsversuche am Steuerrad, das Ruder in Richtung Senkung der $\mathrm{CO}_{2}$-Konzentration einzuschlagen. Jedoch sind diese Kräfte in Summe noch zu schwach, sodass die MS Klima den Kurs weiter Richtung globaler Erwärmung hält. Zudem geben einige Akteure am Kontrollpult auf der Brücke - zum Teil unbewusst - zusätzlich Schub auf das Bugstrahlruder $\mathrm{CH}_{4}$-Konzentration. Unterhalb der Wasserlinie wird die MS Klima so noch stärker in Richtung globale Erwärmung gedrückt.

Die Lehre aus dem Bild der MS Klima lautet, dass sich die Erderwärmung nicht stoppen, sondern lediglich verlangsamen lässt, selbst wenn die Menschheit ab morgen weltweit kein $\mathrm{CO}_{2}$ mehr ausstoßen würde.

Zur Aufnahme des zusätzlich in die Atmosphäre emittierten $\mathrm{CO}_{2}$ gibt es natürliche Kohlenstoffsenken, die in den Beiträgen „Wie Klima funktioniert und warum sich die Atmosphäre erwärmt" sowie "Vom Klimagas zum Wertstoff: $\mathrm{CO}_{2}$ " näher beschrieben werden. Diese Senken bestehen primär aus den Ozeanen und der Vegetation an Land. Zusammen haben sie ein jährliches Fassungsvermögen zwischen zwei und vier Gigatonnen Kohlenstoff, wobei die Angaben hierzu stark schwanken und von den zugrundeliegenden Modellannahmen abhängen. Wenn man aber weiß, dass weltweit durch die Verbrennung von fossilen Brennstoffen im Jahr 2006 etwa 6,7 Gigatonnen Kohlenstoff ausgestoßen wurden und der Betrag für das Jahr 2018 um 10 Gigatonnen liegt und für 2019 sogar höher angenommen wurde (Friedlingstein et al. 2019), offenbart sich das eigentliche Problem. Das Fassungsvermögen der natürlichen Senken war im Jahr 2006 geschätzt nur etwa halb so groß wie die jährlichen $\mathrm{CO}_{2}$-Emissionen durch die Menschen. Auch unter der Annahme, dass das Fassungsvermögen der Kohlenstoffsenken trotz der kontinuierlichen Zerstörung von Vegetation konstant geblieben ist, sind im Jahr 2019 mehr als sieben Gigatonnen Kohlenstoff zusätzlich in die Atmosphäre eingebracht worden. Selbst wenn die

9 Bezogen auf das Temperaturniveau von 1850 bis 1900. 
durch Menschen verursachten $\mathrm{CO}_{2}$-Emissionen ab sofort nicht mehr weiter steigen und gleichzeitig noch um 50 Prozent ihres Wertes reduziert würden, erhöht sich die $\mathrm{CO}_{2}$-Konzentration in der Atmosphäre kontinuierlich, da jährlich immer noch zwei bis drei Gigatonnen Kohlenstoff zusätzlich in die Atmosphäre eingebracht würden.

Um die Erderwärmung zu stoppen und wieder ein stabiles Klima zu erreichen, zum Beispiel bei einer Konzentration um 280 ppm CO 2 , müssen Maßnahmen ergriffen werden, die mehr als 25 Prozent des aktuell vorhandenen $\mathrm{CO}_{2}$ wie auch alle zusätzlich entstehenden $\mathrm{CO}_{2}$-Emissionen aus der Atmosphäre entziehen. Dies gilt sowohl für die von Menschen verursachten als auch die aus natürlichen Quellen freigesetzten $\mathrm{CO}_{2}$-Emissionen.

\section{Handlungsansätze}

Die beschleunigte globale Erwärmung ist eine Tatsache, mit der die Menschheit leben muss. Das ist so banal wie brutal. Aber wie kann man sich dieser Situation stellen, ohne zu verzweifeln? Ähnlich wie bei der Diagnose einer schweren Erkrankung hilft es, weder in Panik und Hysterie zu verfallen noch die Fakten zu ignorieren: Am Ende muss man sein Leben mit dem Befund weiterleben. Dies mag vielleicht sogar mit erheblichen Einschränkungen für den Rest des Lebens einhergehen, aber man kann versuchen, das Leben unter den gegebenen Rahmenbedingungen so gut wie möglich zu gestalten, um Lebensqualität zu wahren.

Hier die knappe Diagnose: Da sich der Entwicklungszeitraum für ein optimistisches Zwei-Grad-Celsius-Ziel-Szenario für Deutschland und wohl auch viele andere europäische Länder von einem Jahrhundert auf ein Jahrzehnt verkürzt hat, werden die meisten der heute lebenden Menschen in den nächsten Jahren den Übergang in eine global um zwei Grad wärmere Umwelt miterleben. Im günstigsten Fall werden sie bis an ihr Lebensende in einer um zwei Grad wärmeren Atmosphäre leben. Im ungünstigen Fall werden sie Zeitzeugen des Übergangs in eine global um drei Grad wärmere Atmosphäre sein. Sowohl das Zwei-Grad-Celsius- als auch das Drei-Grad-CelsiusSzenario machen Anpassungen im Lebenswandel erforderlich, die Einschränkungen und Verhaltensänderungen bedingen. Einige Anpassungen werden eher abrupt erfolgen, wie aufgrund der Corona-Pandemie im Jahr 2020, andere vielleicht über mehrere Jahre gestreckt, wie für die seit 2001 nachweislich immer wärmer werdenden Sommer in Mitteleuropa.

Es stellt sich nicht mehr die Frage, wie der Klimawandel verursacht wurde, sondern wie man mit ihm und in ihm leben wird. Vor diesem Hintergrund muss die Frage beantwortet werden, ob und welche der 17 Ziele der nachhaltigen Entwicklung (SDG) mit ihren Unterzielen überhaupt noch bis 2030 erreichbar sind. Die SDG sollten für die kommenden Jahre die Maxime des globalen Handelns sein, da eine Abkehr 
von ihnen nur das durch die Erderwärmung bedingte Konfliktpotenzial erhöht. Die Überprüfung und Anpassung der SDG mag ein guter Ausgangspunkt für ein abgestimmtes und vereintes globales Handeln sein. Die Reduktion von $\mathrm{CO}_{2}$-Emissionen wie auch die $\mathrm{CO}_{2}$-Abscheidung und Klimaanpassungsmaßnahmen werden in den nächsten Jahren die Aktivitäten sein, welche es uns Menschen erlauben, mit einer stabilen Lebensqualität weiterzuleben.

Die zentrale Klimafrage für die kommenden Jahre lautet: Wie schnell wird die globale Erwärmung zunehmen und kann man verhindern, dass in naher Zukunft globale Kipp-Punkte ausgelöst werden?

Parallel ist die Frage zu klären, ob es möglich sein wird, ohne zusätzliche $\mathrm{CO}_{2}$-Einträge in die Atmosphäre global zu einem stabilen Kohlenstoffkreislauf zurückzukehren. Hauptverursacher der durch Menschen erzeugten $\mathrm{CO}_{2}$-Emissionen ist immer noch die Energiewandlung durch die Verbrennung fossiler Energieträger. Prof. Dr. Robert Schlögel, Direktor des Max-Planck-Instituts für Chemische Energiekonversion und Mitglied der Deutschen Akademie der Technikwissenschaften, sagt: „Klimaschutz und Energieversorgung sind zwei Seiten derselben Medaille." (ARD-alpha 2020) Denn mit den vorhandenen Technologien ist eine $\mathrm{CO}_{2}$-freie Energieerzeugung zur Deckung des globalen Bedarfs kaum umsetzbar. Schlögel fordert daher, dass sich die Politik statt verschiedener zersplitterter Teilziele auf ein Ziel festlegen soll: "Wir nehmen endlich die fossilen Energieträger aus dem [Energie]System raus." (ARD-alpha 2020) Wie kann eine zukünftige Energieversorgung aus heutiger Sicht aussehen? Der Antwort zu den Herausforderungen wird am Beispiel Deutschlands im Kap. 8 „Herausforderungen einer klimafreundlichen Energieversorgung" nachgegangen.

Schaut man sich global die Hauptemittenten von $\mathrm{CO}_{2}$-Emissionen an, so sind die Emissionen der Industrieländer eher konstant und rückläufig. Stattdessen sind asiatische Länder, insbesondere China und Indien, inzwischen die größten $\mathrm{CO}_{2}$-Emittenten, deren Ausstoß in den vergangenen Jahren noch angewachsen ist (siehe Abb. 1.4). Wie sich China und andere Länder durch Kooperationen im Rahmen des Green Deal der Europäischen Kommission stärker bei der Reduktion der $\mathrm{CO}_{2}$-Emissionen einbinden lassen, wird im Kap. 4 „European Green Deal: Hebel für internationale Klima- und Wirtschaftsallianzen" diskutiert.

Die veränderten Rahmenbedingungen unter einer beschleunigten Erderwärmung werden dazu zwingen, insgesamt ressourcenschonender zu wirtschaften. Ein Ansatz hierzu ist die Kreislauf-Wirtschaft, die ausführlicher in Kap. 10 „Digitalisierung - Segen oder Fluch für den Klimaschutz?" und Kap. 13 „Kreislaufwirtschaft als Säule des EU Green Deal" beleuchtet wird. 


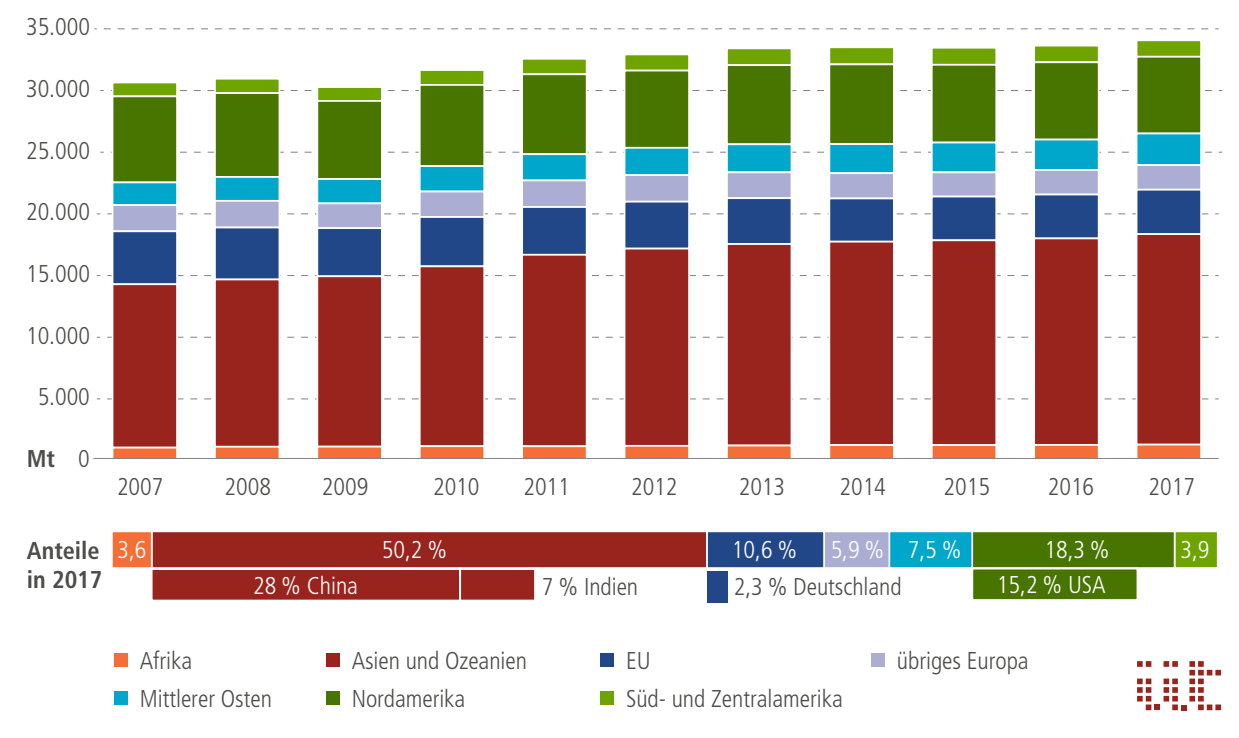

Abb. 1.4 Entwicklung energiebedingter $\mathrm{CO}_{2}$-Emissionen weltweit nach Regionen. Oben im Bild sind die jährlichen globalen $\mathrm{CO}_{2}$-Emissionen in Megatonnen (Mt) für die verschiedenen Regionen dargestellt. Unten sind die Anteile aus dem Jahr 2017 für Regionen und ausgewäh/te Länder angegeben. (Eigene Darstellung nach BP 2018)

Unabhängig davon, was man in Zukunft plant, sei es der jährliche Geschäftsplan, ein Forschungsprojekt oder eine neue kommunale Organisationsstruktur - man wird Projekte wesentlich agiler planen und umsetzen müssen als bisher üblich. Ein Fahren auf Nahsicht wird die neue Projektplanungsmaxime. Es werden sich auch neue Standards bei der Planung etablieren. So werden bei Festlegung von Strategien zur Erreichung mittelfristiger Ziele Foresight-Methoden stärker zum Einsatz kommen. Eine Einführung hierzu wird im Kap. 11 „Zukunft unter Klima-Unsicherheiten agil und nachhaltig gestalten" gegeben.

Dass die Entwicklung des Klimas inzwischen auch die Finanzwirtschaft im erheblichen Umfang bewegt, zeigt ein Interview mit Joachim Wenning, dem Vorstandschef der Münchener RE, vom Dezember 2019: „Wir werden auf allen Kontinenten Folgen der Klimaveränderung erleben - etwa in Form von Stürmen, Überschwemmungen oder Dürren. Die Zahl der Naturkatastrophen wird zunehmen, gleichzeitig wird die Intensität bestimmter Katastrophen extremer. [...] Die Erde darf sich im Vergleich zum vorindustriellen Zeitalter auf keinen Fall um mehr als zwei Grad erwärmen, besser noch um maximal 1,5 Grad. [...] Es wäre wünschenswert, weit vor 2050 komplett auf fossile Energieträger zu verzichten. Wir müssen realistisch bleiben, das wird sehr 
schwierig [...] Für mich ist die entscheidende Frage: Was ist bis dahin tatsächlich machbar? Das muss dann aber auch gemacht werden. [...] Eine wirksame Klimapolitik muss auch weh tun. Bis zum letzten Jahr gab es für die Versicherung von fossilen Energien keine Einschränkungen. [...] Neue Kohlekraftwerke oder Kohleminen versichern wir im Einzelrisikogeschäft seit 2018 nicht mehr [...] Besser für alle ist, wenn technologischer Fortschritt in der erneuerbaren Energieversorgung einen dramatischen Klimawandel vermeidet. [...]" (Merkur 2019)

Vor dem Hintergrund müssen Lösungen zu einer Reduktion bis hin zur Vermeidung von $\mathrm{CO}_{2}$-Emissionen bei der Produktion gefunden werden. Welche Ansätze hierzu existieren und in naher Zukunft einsetzbar sind, wird im Kap. 9 „Wie Industrieproduktion nachhaltig gestaltet werden kann" gezeigt. Die Digitalisierung als Querschnittstechnik hat schon Prozesse und Abläufe in allen Wirtschafts- und Gesellschaftsbereichen radikal verändern können. Kann sie auch dabei helfen, $\mathrm{CO}_{2}$ Reduktionen zu optimieren und zu beschleunigen? Erste Antworten für eine weiterführende Diskussion werden im Kap. 10 „Digitalisierung - Segen oder Fluch für den Klimaschutz?" gegeben.

Die Vermeidung von $\mathrm{CO}_{2}$-Emissionen wird auch den Mobilitätssektor verändern, in dem Antworten auf zwei wesentliche Fragen gefunden werden müssen: Welche neuen Antriebskonzepte können bis wann welches $\mathrm{CO}_{2}$-Einsparpotenzial realisieren? Und welche neuen Mobilitätskonzepte können bis wann welches $\mathrm{CO}_{2}$-Einsparpotenzial realisieren? Lösungsansätze stellt das Kap. 7 "Auf dem Weg zu einer nachhaltigen Mobilität" vor.

Wenn auch die Bedürfnisse und Erwartungen der Menschen sich nicht grundsätzlich von denen in der Vergangenheit unterscheiden werden, so wird doch wie schon in der ersten Phase der Corona-Pandemie im Jahr 2020 augenfällig, eine neue Art der Sensibilisierung für die Versorgung ebenso wie auch für den Schutz vor Unwägbarkeiten in den Alltag zurückkehren. Die Erwartung von Bürgerinnen und Bürger an den Staat werden zunehmen. Es geht um

- die Sicherung der (Grund)Versorgung und den Schutz des Lebensraums,

- die Versorgung mit Wasser, Nahrung, Energie, Medizin und Bildung,

- den Schutz vor Katastrophen, Seuchen und anderem Ungemach,

- die Sicherung von Arbeits- und Erwerbsplätzen,

- die Sicherung von (Wohn)Eigentum.

Das alles wird die staatlichen Institutionen auch auf regionaler und lokaler Ebene herausfordern. Entscheider:innen müssen sich Fragen stellen wie diese: 
- Wie kann man die ansässige Wirtschaft bei den bevorstehenden Herausforderungen stärken und unterstützen?

- Wie stellt man die Versorgung im Rahmen der Veränderungen sicher?

- Wie organisiert man den Schutz für die regelmäßig auftretenden Extremsituationen?

- Wie bewältigt man dies vor dem Hintergrund des demografischen Wandels?

Nicht nur Entscheider:innen in Wirtschaft und Politik, sondern alle Bürger:innen werden sich in den kommenden Jahren auf die anstehenden Auswirkungen der Erderwärmung einstellen müssen. Dazu zählen neben einer steigenden Anzahl extremer Wetterereignisse auch mögliche Einschränkungen von bisher selbstverständlichen Angeboten etwa von Lebensmitteln aus fernen Ländern sowie Reiseangeboten. Wie sich das Bewusstsein und damit das Verhalten ändern lässt, zeigt Kap. 12 "Anders denken und handeln - Bewusstsein für das Klima" auf. Welches Wissen für veränderte Anforderungen in der Ausbildung derzeit vermittelt wird und notwendig ist, wird am Beispiel Deutschland in Kap. 14 „Fridays for Education: Status quo der Nachhaltigkeitsvermittlung in Deutschland" diskutiert.

Mit dem in diesem Buch behandelten Themenkreis ist die Diskussion der durch die beschleunigte Erderwärmung verursachten Veränderungen selbstverständlich nicht abgeschlossen, allerdings in einer Breite eröffnet, die durch mutige Entscheidungen und zeitnahes Handeln eine Zukunft mit nachhaltigen Lebensbedingungen für alle möglich macht. 


\section{Literatur}

ARD-alpha (2020): Keine Energiewende ohne Katalyse, Campus Talks vom 13.01.2020.

Online verfügbar unter https://www.ardmediathek.de/ard/player/Y3JpZDovL2JyLmRIL3Z pZGVvLzlkZjZiNGM1LTQyNDMtNDdhMy1hOThjLTdmYjcwMzc2YjBhOQ/keine-energiewende-ohne-katalyse, zuletzt geprüft am 17.07.2020.

Battisti, David S.; Naylor, Rosamund L. (2009): Historical Warnings of Future Food Insecurity with Unprecedented Seasonal Heat, Science 323, Nr. 5911; Januar 2009, Seite 204-244.

Beerling, David J. et al. (2020): Potential for large-scale $\mathrm{CO}_{2}$ removal via enhanced rock weathering with croplands, Nature volume 583, Seite 242-248 (2020), DOI: https:// doi.org/10.1038/s41586-020-2448-9. Online verfügbar unter https://www.nature.com/ articles/s41586-020-2448-9, zuletzt geprüft am 17.07.2020.

BP (2018): BP Statistical Review of World Energy, Juni 2018, Seite 49. Online verfügbar unter https://www.bp.com/content/dam/bp/business-sites/en/global/corporate/pdfs/energyeconomics/statistical-review/bp-stats-review-2018-full-report.pdf, zuletzt geprüft am 17.07.2020.

Bundesregierung (2020): Globale Nachhaltigkeitsstrategie - Nachhaltigkeitsziele verständlich erklärt. Online verfügbar unter https://www.bundesregierung.de/breg-de/themen/nachhaltigkeitspolitik/nachhaltigkeitsziele-verstaendlich-erklaert-232174, zuletzt geprüft am 14.08.2020.

Climate Action Tracker (2019): The CAT Thermometer. Online verfügbar unter https://climateactiontracker.org/global/cat-thermometer/, zuletzt geprüft am 03.07.2020.

Commonwealth of Australia (2020): Estimating greenhouse gas emissions from bushfires in Australia's temperate forests: focus on 2019-20, April 2020, Seite 9. Online verfügbar unter https://www.industry.gov.au/sites/default/files/2020-04/estimating-greenhouse-gasemissions-from-bushfires-in-australias-temperate-forests-focus-on-2019-20.pdf, zuletzt geprüft am 17.07.2020.

DWD (2020): Basisklimavorhersagen: 1-10 Jahre. 11.03.2020. Online verfügbar unter https://www.dwd.de/DE/leistungen/klimavorhersagen/_node_basis_jahre.html, zuletzt geprüft am 03.07.2020.

Faust, Eberhard; Rauch, Ernst (2020): Serie von heißen Jahren und mehr Wetterextreme (02.03.2020). Online verfügbar unter https://www. munichre.com/topics-online/de/ climate-change-and-natural-disasters/climate-change/climate-change-heat-records-andextreme-weather.html, zuletzt geprüft am 18.07.2020.

Friedlingstein, Pierre; Jones, Matthew W.; et al. (2019): Global Carbon Budget 2019, Syst. Sci. Data, 11, 1783-1838, 2019, DOI https://doi.org/10.5194/essd-11-1783-2019. Online verfügbar unter https://essd.copernicus.org/articles/11/1783/2019/, zuletzt geprüft am 26.07.2020.

Geden, Oliver; Schenuit, Felix (2020): Unkonventioneller Klimaschutz - Gezielte $\mathrm{CO}_{2}$-Entnahme aus der Atmosphäre als neuer Ansatz in der EU-Klimapolitik, SWP Stiftung Wissenschaft und Politik Deutsches Institut für Internationale Politik und Sicherheit, Mai 
2020, ISSN 1611-6372. Online verfügbar unter https://www.swp-berlin.org/fileadmin/ contents/products/studien/2020S10_Gdn_Schenuit_CO2Entnahme.pdf, zuletzt geprüft am 17.07.2020.

Graver, Brandon; Zhang, Kevin; Rutherford, Dan (2019): $\mathrm{CO}_{2}$ emissions from commercial aviation, 2018, ICCT, September 2019. Online verfügbar unter https://theicct.org/publications/co2-emissions-commercial-aviation-2018, zuletzt geprüft am 17.07.2020.

IPCC (2014): Klimaänderung 2014: Synthesebericht. Beitrag der Arbeitsgruppen I, II und III zum Fünften Sachstandsbericht des Zwischenstaatlichen Ausschusses für Klimaänderungen (IPCC) [Hauptautoren, Rajendra K. Pachauri und Leo A. Meyer (Hrsg.)]. IPCC, Genf, Schweiz. Deutsche Übersetzung durch Deutsche IPCC-Koordinierungsstelle, Bonn, 2016. Online verfügbar unter https://www.de-ipcc.de/media/content/IPCC-AR5_SYR_barrierefrei.pdf, zuletzt geprüft am 03.07.2020.

IPCC (2018): 1,5 ${ }^{\circ} \mathrm{C}$ GLOBALE ERWÄRMUNG Zusammenfassung für politische Entscheidungsträger, ISBN: 978-3-89100-051-9, Deutsche Übersetzung durch Deutsche IPCCKoordinierungsstelle, Bonn, 2018. Online verfügbar unter https://www.ipcc.ch/site/assets/ uploads/2019/03/SR1.5-SPM_de_barrierefrei-2.pdf, zuletzt geprüft am 03.07.2020.

Jackson, Robert B.; Saunois, Marielle; Bousquet, Philippe; Canadell, Josep G.; Poulter, Benjamin; Stavert, Ann R; Bergamaschi, Peter; Niwa, Y; Segers, Arjo; Tsuruta, Aki (2020): Increasing anthropogenic methane emissions arise equally from agricultural and fossil fuel sources, Environmental Research Letters 2020, DOI: https://doi.org/10.1088/1748-9326. Online verfügbar unter https://drive.google.com/file/d/19jfjwXqDfLdARtvcHGfdpYcVOkxPIpV/view, zuletzt geprüft am 17.07.2020.

Jalili, Paria; Saydam, Serkan; Cinar, Yildiray (2011): $\mathrm{CO}_{2}$ Storage in Abandoned Coal Mines, 2011 Underground Coal Operators' Conference, 10 und 11 Februar 2011, S. 355. Online verfügbar unter https://ro.uow.edu.au/cgi/viewcontent.cgi?article=2039\&context=coal, zuletzt geprüft am 03.07.2020.

Merkur (2019): Rückversicherer Munich Re warnt vor Klimawandel - „Klimaschutz muss weh tun im Geldbeutel“, vom 20. Dezember 2019. Online verfügbar unter https://www. merkur.de/wirtschaft/muenchen-klimawandel-rueckversicherer-munich-re-warnt-folgenenergie-katastrophen-13356307.html, zuletzt geprüft am 18.07.2020.

Saunois, Marielle; Stavert, Ann R; Poulter, Ben; Bousquet, Philippe; Canadell, Josep G; Jackson, Robert B; et al. (2020): The Global Methane Budget 2000-2017, Earth Syst. Sci. Data, 12, 1561-1623, 2020, DOI: https://doi.org/10.5194/essd-12-1561-2020. Online verfügbar unter https://essd.copernicus.org/articles/12/1561/2020/, zuletzt geprüft am 17.07.2020.

Tagesschau (2020): Rekordmenge an Methan heizt Klima auf, vom 15.07.2020. Online verfügbar unter https://www.tagesschau.de/ausland/methan-treibhauswirkung-studie-101. html, zuletzt geprüft am 17.07.2020.

Umweltbundesamt (2020a): Trends der Lufttemperatur. 23.03.2020. Online verfügbar unter https://www.umweltbundesamt.de/daten/klima/trends-der-lufttemperatur, zuletzt geprüft am 09.07.2020. 
Umweltbundesamt (2020b): Atmosphärische Treibhausgas-Konzentrationen. 24.06.2020. Online verfügbar unter https://www.umweltbundesamt.de/daten/klima/atmosphaerischetreibhausgas-konzentrationen, zuletzt geprüft am 03.07.2020.

United Nations (2015): Sustainable Development Goals. Online verfügbar unter https://sustainabledevelopment.un.org, zuletzt geprüft am 03.07.2020.

Wallace-Wells, David (2019): Die unbewohnbare Erde - Leben nach der Erderwärmung, Ludwig Verlag München, ISBN 978-3-453-28118-9.

World Health Organization (2017): Protecting Health in Europe from Climate Change: 2017 Update, World Health Organization 2017, ISBN 9789289052832. Online verfügbar unter https://www.euro.who.int/_data/assets/pdf_file/0004/355792/ProtectingHealthEuropeFromClimateChange.pdf?ua=1, zuletzt geprüft am 05.07.2020.

WMO (2020a): WMO confirms 2019 as second hottest year on record, 25. 01.2020. Online verfügbar https://public.wmo.int/en/media/press-release/wmo-confirms-2019-secondhottest-year-record, zuletzt geprüft am 29.07.2020.

WMO (2020b): New climate predictions assess global temperatures in coming five years, 08.07.2020. Online verfügbar unter https://public.wmo.int/en/media/press-release/newclimate-predictions-assess-global-temperatures-coming-five-years, zuletzt geprüft am 29.07.2020.

Zeit (2020): Weltweiter Methanausstoß steigt auf Rekordwert, ZEIT ONLINE, dpa, vom 15.07.2020. Online verfügbar unter https://www.zeit.de/wissen/umwelt/2020-07/treibhausgas-methan-klimawandel-us-studien-corona-krise, zuletzt geprüft am 26.07.2020.

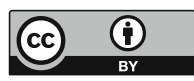

Dieses Kapitel wird unter der Creative Commons Namensnennung 4.0 International Lizenz http://creativecommons.org/licenses/by/4.0/deed.de) veröffentlicht, welche die Nutzung, Vervielfältigung, Bearbeitung, Verbreitung und Wiedergabe in jeglichem Medium und Format erlaubt, sofern Sie den/die ursprünglichen Autor(en) und die Quelle ordnungsgemäß nennen, einen Link zur Creative Commons Lizenz beifügen und angeben, ob Änderungen vorgenommen wurden.

Die in diesem Kapitel enthaltenen Bilder und sonstiges Drittmaterial unterliegen ebenfalls der genannten Creative Commons Lizenz, sofern sich aus der Abbildungslegende nichts anderes ergibt. Sofern das betreffende Material nicht unter der genannten Creative Commons Lizenz steht und die betreffende Handlung nicht nach gesetzlichen Vorschriften erlaubt ist, ist für die oben aufgeführten Weiterverwendungen des Materials die Einwilligung des jeweiligen Rechteinhabers einzuholen. 